\title{
ON FINITE DIFFERENCE INEQUALITY OF LYAPUNOV TYPE
}

\author{
GOU-SHENG YANG, SHIOW-FU HUANG
}

\section{Introduction}

The classical inequality of Lyapunov [1] states that if $y(t)$ is a nontrivial solution of the differential system

$$
\begin{aligned}
& y^{\prime \prime}(t)+p(t) y(t)=0, \quad a \leq t \leq b, \\
& y(a)=y(b)=0,
\end{aligned}
$$

where $p(t)$ is a real and continuous function defined on $[\mathrm{a}, \mathrm{b}]$, then

$$
(b-a) \int_{a}^{b}|p(t)| d t>4 .
$$

Write throughout $\Delta x(n)=x(n+1)-x(n)$.

Pachaptte has establihed in [2] the following discrete analogue of Lyapunov type inequality:

Theorem $\mathbb{A}$. Let $p(n)$ and $r(n)$ be real-valued functions defined on $I=$ $\{a, a+1, a+2 . \cdots, b\}$, where $a, b$ are integers, and $r(n)>0$ for $n \in I$. If $x(n)$ is a solution of the equation

$$
\Delta(r(n) \Delta x(n))+p(n) x(n)=0,
$$

such that $x(a)=x(b)=0$, and $x(n) \neq 0, \forall n \in I^{0}=\{a+1, a+2, \cdots, b-1\}$, then

$$
4 \leq\left(\sum_{n=a}^{b-1} \frac{1}{r(n)}\right)\left(\sum_{n=a}^{b-2}|p(n)|\right) .
$$


The main purpose of this paper is to given some generalizations of Theorem A.

\section{Preliminary Results}

In order to prove our main results, we need the follwoing lemmas:

Lemma 1. If $u, v$ are any functions defined on $I$, and $m$ is a positive integer then

$$
\sum_{n=a}^{b-1} u^{m}(n) \Delta v(n)=u^{m}(b-1) v(b)-u^{m}(a) v(a)-\sum_{n=a}^{b-2} v(n+1) \Delta u(n) w(n)
$$

where $w(n)=u^{m-1}(n+1)+u^{m-2}(n+1) u(n)+\cdots+u(n+1) u^{m-2}(n)+u^{m-1}(n)$.

Proof. We have

$$
\begin{aligned}
& \sum_{n=a}^{b-1} u^{m}(n) \Delta v(n) \\
= & \sum_{n=a}^{b-1} u^{m}(n)(v(n+1)-v(n)) \\
= & \sum_{n=a}^{b-1} u^{m}(n) v(n+1)-\sum_{n=a}^{b-1} u^{m}(n) v(n) \\
= & \sum_{n=a}^{b-1} u^{m}(n) v(n+1)-\sum_{n=a-1}^{b-2} u^{m}(n+1) v(n+1) \\
= & u^{m}(b-1) v(b)-u^{m}(a) v(a)-\sum_{n=a}^{b-2}\left(u^{m}(n+1)-u^{m}(n)\right) v(n+1) .
\end{aligned}
$$

Since

$$
\begin{aligned}
& u^{m}(n+1)-u^{m}(n) \\
= & (u(n+1)-u(n))\left(u^{m-1}(n+1)+u^{m-2}(n+1) u(n)+\cdots\right. \\
& \left.+u(n+1) u^{m-2}(n)+u^{m-1}(n)\right) \\
= & \Delta u(n) w(n) .
\end{aligned}
$$


it follows that

$$
\sum_{n=a}^{b-1} u^{m}(n) \Delta v(n)=u^{m}(b-1) v(b)-u^{m}(a) v(a)-\sum_{n=a}^{b-2} v(n+1) \Delta u(n) w(n) .
$$

Lemma 2. Under the hypotheses of lemma 1, if $u(n)=r(n) \Delta x(n)$, and $M=\max _{k \in 1}|x(k)|$, then

$$
|w(n)| \leq(2 M)^{m-1} \sum_{i=1}^{m} r^{m-i}(n+1) r^{i-1}(n) .
$$

Proof. We have

$$
\begin{aligned}
w(n) & =u^{m-1}(n+1)+u^{m-2}(n+1) u(n)+\cdots+u(n+1) u^{m-2}(n)+u^{m-1}(n) \\
& =\sum_{i=1}^{m} u^{m-i}(n+1) u^{i-1}(n) \\
& =\sum_{i=1}^{m}(r(n+1) \Delta x(n+1))^{m-i}(r(n) \Delta x(n))^{i-1}
\end{aligned}
$$

so that

$$
\begin{aligned}
|w(n)| & \leq \sum_{i=1}^{m} r^{m-i}(n+1)|\Delta x(n+1)|^{m-i} r^{i-1}(n)|\Delta x(n)|^{i-1} \\
& \leq(2 M)^{m-1} \sum_{i=1}^{m} r^{m-i}(n+1) r^{i-1}(n) .
\end{aligned}
$$

\section{Main Results}

Theorem 1. Let $x(n)$ be a solution of the equation (1) such that $x(a)=$ $x(b)=0$, and $x(n) \neq 0, \forall n \in I^{0}$. If $m$ is a nonnegative integer, then

$$
4 \leq\left(\sum_{n=a}^{b-1} \frac{1}{r(n)}\right)^{2 m+1}\left(\sum_{n=a}^{b-2}|p(n)| \sum_{i=1}^{2 m+1} r^{2 m+1-i}(n+1) r^{i-1}(n)\right) .
$$


Proof. Let $M=\max _{k \in I^{\circ}}|x(k)|$. Then $M>0$, since $x(n) \neq 0, \forall n \in I^{0}$. From the following identities:

$$
\begin{aligned}
& x(k)=\sum_{n=a}^{k-1} \Delta x(n), \quad k \in I^{0} . \\
& x(k)=-\sum_{n=k}^{b-1} \Delta x(n), \quad k \in I^{0},
\end{aligned}
$$

it follows that

$$
2|x(k)| \leq \sum_{n=a}^{b-1}|\Delta x(n)|, \quad k \in I^{0} .
$$

Hence

$$
2 M \leq \sum_{n=a}^{b-1}|\Delta x(n)|
$$

so that

$$
(2 M)^{2 m+2} \leq\left(\sum_{n=a}^{b-1} r^{\frac{-2 m-1}{2 m+2}}(n) r^{\frac{2 m+1}{2 m+2}}(n)|\Delta x(n)|\right)^{2 m+2} .
$$

Using Hölder's inequality with indices $\frac{2 m+2}{2 m+1}, 2 m+2$ we have

$$
\begin{aligned}
(2 M)^{2 m+2} & \leq\left(\sum_{n=a}^{b-1}\left(r^{\frac{-2 m-1}{2 m+2}}(n)\right)^{\frac{2 m+2}{2 m+1}}\right)^{2 m+1}\left(\sum_{n=a}^{b-1}\left(r^{\frac{2 m+1}{2 m+2}}(n)|\Delta x(n)|\right)^{2 m+2}\right) \\
& =\left(\sum_{n=a}^{b-1} \frac{1}{r(n)}\right)^{2 m+1}\left(\sum_{n=a}^{b-1} r^{2 m+1}(n)|\Delta x(n)|^{2 m+2}\right) \\
& =\left(\sum_{n=a}^{b-1} \frac{1}{r(n)}\right)^{2 m+1}\left(\sum_{n=a}^{b-1}(r(n) \Delta x(n))^{2 m+1} \Delta x(n)\right) .
\end{aligned}
$$

It follows from Lemma 1 that

$$
(2 M)^{2 m+2} \leq\left(\sum_{n=a}^{b-1} \frac{1}{r(n)}\right)^{2 m+1}\left(-\sum_{n=a}^{b-2}(x(n+1) \Delta(r(n) \Delta x(n)) w(n))\right),
$$

where $w(n)=\sum_{i=o}^{2 m}(r(n+1) \Delta x(n+1))^{2 m-i}(r(n) \Delta x(n))^{i}$, Since $\Delta(r(n) \Delta x(n))=$ 
$-p(n) x(n)$, it follows from (4), and lemma 2 that

$$
\begin{aligned}
(2 M)^{2 m+2} & \leq\left(\sum_{n=a}^{b-1} \frac{1}{r(n)}\right)^{2 m+1}\left(\sum_{n=a}^{b-2} x(n+1) p(n) x(n) w(n)\right) \\
& \leq\left(\sum_{n=a}^{b-1} \frac{1}{r(n)}\right)^{2 m+1}\left(\sum_{n=a}^{b-2}|x(n+1)\|p(n)\| x(n) \| w(n)|\right) \\
& \leq\left(\sum_{n=a}^{b-1} \frac{1}{r(n)}\right)^{2 m+1} M^{2}\left(\sum_{n=a}^{b-2}|p(n) \| w(n)|\right) \\
& \leq\left(\sum_{n=a}^{b-1} \frac{1}{r(n)}\right)^{2 m+1} M^{2}\left(\sum_{n=a}^{b-2}|p(n)|(2 M)^{2 m} \sum_{i=1}^{2 m+1} r^{2 m+1-i}(n) r^{i-1}(n)\right) .
\end{aligned}
$$

Therefore

$$
4 \leq\left(\sum_{n=a}^{b-1} \frac{1}{r(n)}\right)^{2 m+1}\left(\sum_{n=a}^{b-2}|p(n)| \sum_{i=1}^{2 m+1} r^{2 m+1-i}(n+1) r^{i-1}(n)\right) .
$$

This completes the proof of the theorem.

Remark 1. In the special case $m=0$, the inequality (3) reduces to the inequality (2) in Theorem A.

Theorem 2. Let $p_{1}(n), p_{2}(n), r(n)$ be real-valued functions defined on $I$, and $r(n)>0$ for $n \in I=\{a, a+1, \cdots, b\}$. Let $x(n)$ be a solution of the equation $\Delta(r(n) \Delta x(n))+p_{1}(n) \Delta x(n)+p_{2}(n) x(n)=0$ such that $x(a)=x(b)=0$ and $x(n) \neq 0, \forall n \in I^{0}$. If $m$ is a nonnegative integer, then

$$
4 \leq\left(\sum_{n=a}^{b-1} \frac{1}{r(n)}\right)^{2 m+1}\left(\sum_{n=a}^{b-2}\left(2\left|p_{1}(n)\right|+\left|p_{2}(n)\right|\right) \sum_{i=1}^{2 m+1} r^{2 m+1-i}(n+1) r^{i-1}(n)\right) .
$$

Proof. As in the proof of theorem 1, we have

$$
(2 M)^{2 m+2} \leq\left(\sum_{n=a}^{b-1} \frac{1}{r(n)}\right)^{2 m+1}\left(-\sum_{n=a}^{b-2} x(n+1) \Delta(r(n) \Delta x(n)) w(n)\right)
$$


Since $-\Delta(r(n) \Delta x(n))=p_{1}(n) \Delta x(n)+p_{2}(n) x(n)$, it follows that

$$
\begin{aligned}
&(2 M)^{2 m+2} \leq\left(\sum_{n=a}^{b-1} \frac{1}{r(n)}\right)^{2 m+1}\left(\sum_{n=a}^{b-2} x(n+1)\left(p_{1}(n) \Delta x(n)+p_{2}(n) x(n)\right) w(n)\right) \\
& \leq\left(\sum_{n=a}^{b-1} \frac{1}{r(n)}\right)^{2 m+1}\left(\sum _ { n = a } ^ { b - 2 } | x ( n + 1 ) | \left(\left|p_{1}(n)\right||\Delta x(n)|\right.\right. \\
&\left.\left.+\left|p_{2}(n)\right||x(n)|\right)|w(n)|\right) \\
& \leq\left(\sum_{n=a}^{b-1} \frac{1}{r(n)}\right)^{2 m+1}\left(\sum_{n=a}^{b-2} M\left(\left|p_{1}(n)\right| 2 M+\left|p_{2}(n)\right| M\right)|w(n)|\right) \\
& \leq\left(\sum_{n=a}^{b-1} \frac{1}{r(n)}\right)^{2 m+1}\left(\sum_{n=a}^{b-2} M^{2}\left(2\left|p_{1}(n)\right|+\left|p_{2}(n)\right|\right)(2 M)^{2 m}\right. \\
&\left.\sum_{i=1}^{2 m+1} r^{2 m+1-i}(n+1) r^{i-1}(n)\right) .
\end{aligned}
$$

Thus

$$
4 \leq\left(\sum_{n=a}^{b-1} \frac{1}{r(n)}\right)^{2 m+1}\left(\sum_{n=a}^{b-2}\left(2\left|p_{1}(n)\right|+\left|p_{2}(n)\right|\right) \sum_{i=1}^{2 m+1} r^{2 m+1-i}(n+1) r^{i-1}(n)\right) .
$$

Remark 2. The inequality (5) reduces to the inequality (3) when $p_{1}(n) \equiv 0$.

Theorem. 3. Let $x(n)$ be a solution of the equation $(1)$ such that $x(a)=$ $\sigma_{1} x(a+1), x(b)=\sigma_{2} x(b-1)$, where $-1 \leq \sigma_{1}, \sigma_{2} \leq 1$, and $x(n) \neq 0 . \forall n \in I^{0}$. If $m$ is a nonnegative integer, then

$$
\begin{aligned}
& \left(2-\left|\sigma_{1}\right|-\left|\sigma_{2}\right|\right)^{2 m+2} \\
& \leq\left(\sum_{n=a}^{b-1} \frac{1}{r(n)}\right)^{2 m+1}\left(r^{2 m+1}(b-1)\left(1-\sigma_{2}\right)^{2 m+1}\left|\sigma_{2}\right|\right. \\
& \left.\quad+r^{2 m+1}(a)\left(1-\sigma_{1}\right)^{2 m+1}\left|\sigma_{1}\right|+\sum_{n=a}^{b-2}|p(n)| 2^{2 m} \sum_{i=1}^{2 m+1} r^{2 m+1-i}(n+1) r^{i-1}(n)\right)
\end{aligned}
$$

Proof. Let $M=\max _{k \in I}|x(k)|$. Then $M>0$, since $x(n) \neq 0, \forall n \in I^{0}$. We have

$$
x(k)=\sum_{n=a}^{k-1} \Delta x(n)+x(a), \text { and } x(k)=x(b)-\sum_{n=k}^{b-1} \Delta x(n)
$$


so that

$$
|x(k)| \leq \sum_{n=a}^{k-1}|\Delta x(n)|+|x(a)| \text { and }|x(k)| \leq x(b)+\sum_{n=k}^{b-1}|\Delta x(n)|
$$

Hence

$$
\begin{aligned}
2|x(k)| & \leq \sum_{n=a}^{b-1}|\Delta x(n)|+|x(a)|+|x(b)| \\
& =\sum_{n=a}^{b-1}|\Delta x(n)|+\left|\sigma_{1}\right||x(a+1)|+\left|\sigma_{2}\right||x(b-1)| \\
& \leq \sum_{n=a}^{b-1}|\Delta x(n)|+\left(\left|\sigma_{1}\right|+\left|\sigma_{2}\right|\right) M, \quad k \in I
\end{aligned}
$$

which gives

$$
2 M \leq \sum_{n=a}^{b-1}|\Delta x(n)|+\left(\left|\sigma_{1}\right|+\left|\sigma_{2}\right|\right) M
$$

On substraction and rise to the power $2 m+2$, where $m>0$, we have

$$
\begin{aligned}
\left(2-\left|\sigma_{1}\right|-\left|\sigma_{2}\right|\right)^{2 m+2} M^{2 m+2} & \leq\left(\sum_{n=a}^{b-1}|\Delta x(n)|\right)^{2 m+2} \\
& =\left(\sum_{n=a}^{b-1} r^{\frac{-2 m-1}{2 m+2}}(n) r^{\frac{2 m+1}{2 m+2}}(n)|\Delta x(n)|\right)^{2 m+2}
\end{aligned}
$$

Using lemma 1, lemma 2 and applying Hölder's inequality to infer

$$
\begin{aligned}
& \left(2-\left|\sigma_{1}\right|-\left|\sigma_{2}\right|\right)^{2 m+2} M^{2 m+2} \\
& \leq\left(\sum_{n=a}^{b-1} \frac{1}{r(n)}\right)^{2 m+1}\left(\sum_{n=a}^{b-1} r^{2 m+1}(n)|\Delta x(n)|^{2 m+2}\right) \\
& =\left(\sum_{n=a}^{b-1} \frac{1}{r(n)}\right)^{2 m+1}\left(\sum_{n=a}^{b-1}(r(n) \Delta x(n))^{2 m+2} \Delta x(n)\right)
\end{aligned}
$$




$$
\begin{aligned}
= & \left(\sum_{n=a}^{b-1} \frac{1}{r(n)}\right)^{2 m+1}\left((r(b-1) \Delta x(b-1))^{2 m+1} x(b)-(r(a) \Delta x(a))^{2 m+1} x(a)\right. \\
& \left.-\sum_{n=a}^{b-2} x(n+1) \Delta(r(n) \Delta x(n)) w(n)\right) \\
= & \left(\sum_{n=a}^{b-1} \frac{1}{r(n)}\right)^{2 m+1}\left((r(b-1) \Delta x(b-1))^{2 m+1} x(b)-(r(a) \Delta x(a))^{2 m+1} x(a)\right. \\
& \left.+\sum_{n=a}^{b-2} x(n+1) p(n) x(n) w(n)\right) \\
\leq & \left(\sum_{n=a}^{b-1} \frac{1}{r(n)}\right)^{2 m+1}\left(r^{2 m+1}(b-1)|\Delta x(b-1)|^{2 m+1}|x(b)|\right. \\
& \left.+r^{2 m+1}(a)|\Delta x(a)|^{2 m+1}|x(a)|+\sum_{n=a}^{b-2}|x(n+1) \| p(n)||x(n)||w(n)|\right) .
\end{aligned}
$$

Since

$$
\begin{aligned}
|\Delta x(b-1)|^{2 m+1} & =|x(b)-x(b-1)|^{2 m+1} \\
& =\left|\sigma_{2} x(b-1)-x(b-1)\right|^{2 m+1} \leq\left|\sigma_{2}-1\right|^{2 m+1} M^{2 m+1} \\
|x(b)| & =\left|\sigma_{2} x(b-1)\right| \leq \sigma_{2} M \\
|\Delta x(a)|^{2 m+1} & =|x(a+1)-x(a)|^{2 m+1} \\
& =\left|x(a+1)-\sigma_{1} x(a+1)\right|^{2 m+1} \leq\left|1-\sigma_{1}\right|^{2 m+1} M^{2 m+1} \\
|x(a)| & =\left|\sigma_{1} x(a+1)\right| \leq\left|\sigma_{1}\right| M,
\end{aligned}
$$

substituting these facts to (7) we have

$$
\begin{aligned}
\left(2-\left|\sigma_{1}\right|-\left|\sigma_{2}\right|\right)^{2 m+2} M^{2 m+2} & \\
\leq & \left(\sum_{n=a}^{b-1} \frac{1}{r(n)}\right)^{2 m+1} \cdot\left(r^{2 m+1}(b-1)\left(1-\sigma_{2}\right)^{2 m+1}\left|\sigma_{2}\right| M^{2 m+2}\right. \\
& \quad+r^{2 m+1}(a)\left(1-\sigma_{1}\right)^{2 m+1}\left|\sigma_{1}\right| M^{2 m+2} \\
& \left.+\sum_{n=a}^{b-2}|p(n)| M^{2}(2 M)^{2 m} \sum_{i=1}^{2 m+1} r^{2 m+1-i}(n+1) r^{i-1}(n)\right) .
\end{aligned}
$$


Dividing both sides by $M^{2 m+2}$ we get the desired inequality.

Remark 3. When $\sigma_{1}=\sigma_{2}=0$, the inequality (6) reduced to the inequality (3).

\section{References}

[1] A. M. Liapunov, "probléme gémérale de la stabilité du mouvement," Annals of Mathematics Strdy 17, Princeton University Press, 1949.

[2] B. G. Pachpatte, "On Lyapunov type finite difference inequality," Tamkang J. Math. 1990, vol. 21, No. 4, 337-339.

Department of Mathematics, Tamkang University, Tamsui, Taiwan 25137. 